\title{
International Archives of Internal Medicine
}

\section{Cutaneous Angiosarcoma in a Psoriasis Patient on Infliximab and Methotrexate Therapy}

\author{
Annika Silfvast-Kaiser ${ }^{1}$, Amanda Abramson Lloyd ${ }^{2}$ and Alan Menter ${ }^{1 *}$ \\ ${ }^{1}$ Division of Dermatology, Baylor University Medical Center, Dallas, TX, USA \\ ${ }^{2}$ Skin and Vein Institute, Encinitas, CA, USA
}

*Corresponding author: Alan Menter, MD, FAAD, Division of Dermatology, Baylor University Medical Center, 3900 Junius St, Suite 145 Dallas, TX 75246, USA, Tel: 972-386-7546

\begin{abstract}
Angiosarcoma is a soft tissue sarcoma of endothelial origin that can arise in any organ, with an incidence of up to $5 \%$ of all malignant cutaneous tumors. It is the $4^{\text {th }}$ most common sarcoma of the skin and typically occurs on the scalp of white men over 60 years of age. Its prognosis is traditionally very poor, with 5-year survival rates reported as low as $11 \%$. Risk factors for cutaneous angiosarcoma have not been clearly established. Herein, we describe a 64-yearold male with a chronic history of psoriasis maintained on methotrexate and infliximab for over 10 years, who presented with a 7-week history of a cluster of reddish-blue, violaceous nodules on his occipital scalp. Biopsy showed classic features of angiosarcoma. He was treated with multimodal therapy. Six years subsequent to his initial diagnosis he continues in remission, with no evident signs of recurrence or further metastases.
\end{abstract}

\section{Keywords}

Cutaneous angiosarcoma, Scalp, Methotrexate, Infliximab, Immunosuppression

\section{Introduction}

Angiosarcoma is a tumor of endothelial cell origin and is considered one of the rarest forms of sarcoma. Primary cutaneous angiosarcoma of the head and neck region is the most common, often insidious with its asymptomatic presentation, initially resembling a benign entity such as a bruise [1]. Its subtlety likely contributes to the difficulty of early diagnosis and the aggressive quality of the tumor at diagnosis. If left untreated, it inevitably progresses to a more thickened nodular lesion which may eventually ulcerate or bleed and can grow to reach a diameter over $20 \mathrm{~cm}[2,3]$. Local control is dif- ficult to achieve and a multifaceted approach to treatment usually achieves better outcomes than monotherapy. Recurrence and metastases are common with very poor survival rates. This is a case of cutaneous angiosarcoma of the scalp that presented in a patient on immunosuppressive therapy for psoriasis. To our knowledge this is the first case report regarding the development of cutaneous angiosarcoma in a patient on immunosuppressive therapy with infliximab and methotrexate (MTX). Whether there is a specific effect of these drugs on the development of the patient's angiosarcoma remains uncertain.

\section{Case Description}

A 64-year-old balding male with history of well-controlled psoriasis maintained on Methotrexate for 12 years (accumulative dose $5630 \mathrm{mg}$ ) and Remicade (infliximab) infusion for 11 years presented with a 7-week history of four "bumps" on his scalp that were initially not bothersome but had begun bleeding a week prior to presentation. He denied any history of skin cancer but did report a moderate history of sun exposure. He also denied any systemic symptoms. On physical exam, 4 adjacent friable, violaceous nodules were seen on the left medial-posterior occipital scalp measuring approximately $5 \mathrm{~cm} \times 3 \mathrm{~cm}$ (Figure $1 \mathrm{~A}$ and Figure 1B). Shave biopsy was consistent with angiosarcoma, showing dermal proliferation of irregular anastomosing vascular channels lined by epithelioid endothelial cells with rare mitoses and prominent cytologic atypia. Hemorrhage was present as well. Atypical cells stained positive for the vascular endothelial markers VEGF, CD34, and

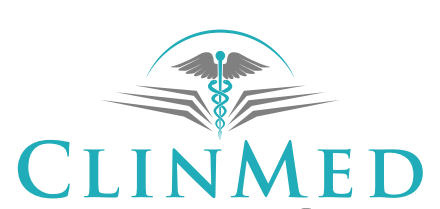

INTERNATIONAL LIBRARY

Citation: Silfvast-Kaiser A, Lloyd AA, Menter A (2018) Cutaneous Angiosarcoma in a Psoriasis Patient on Infliximab and Methotrexate Therapy. Int Arch Intern Med 2:006.

Accepted: October 10, 2018: Published: October 12, 2018

Copyright: (C) 2018 Silfvast-Kaiser A, et al. This is an open-access article distributed under the terms of the Creative Commons Attribution License, which permits unrestricted use, distribution, and reproduction in any medium, provided the original author and source are credited. 


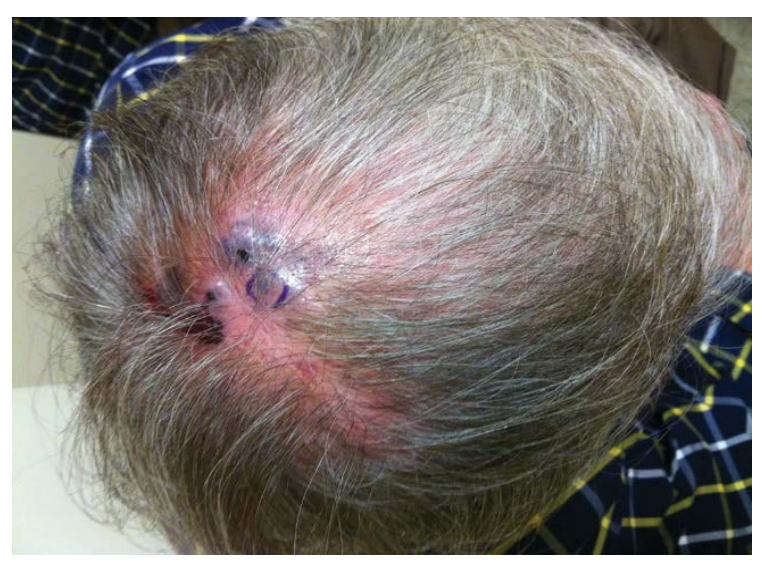

A

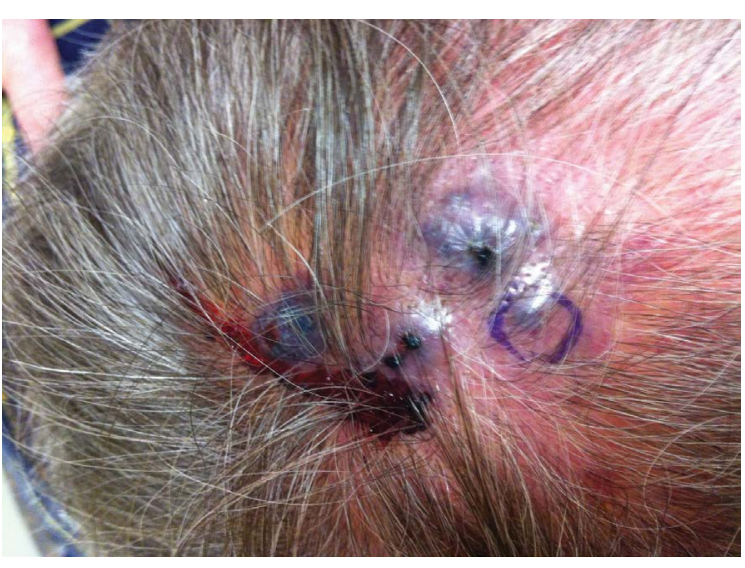

B

Figure 1: A, B) Discrete red-purple nodules on patient's medial-posterior occipital scalp prior to initial diagnostic biopsy.

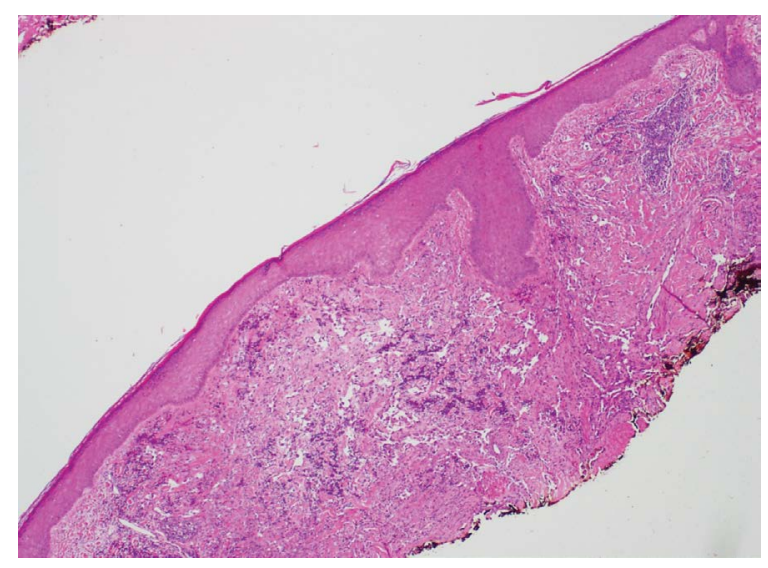

A

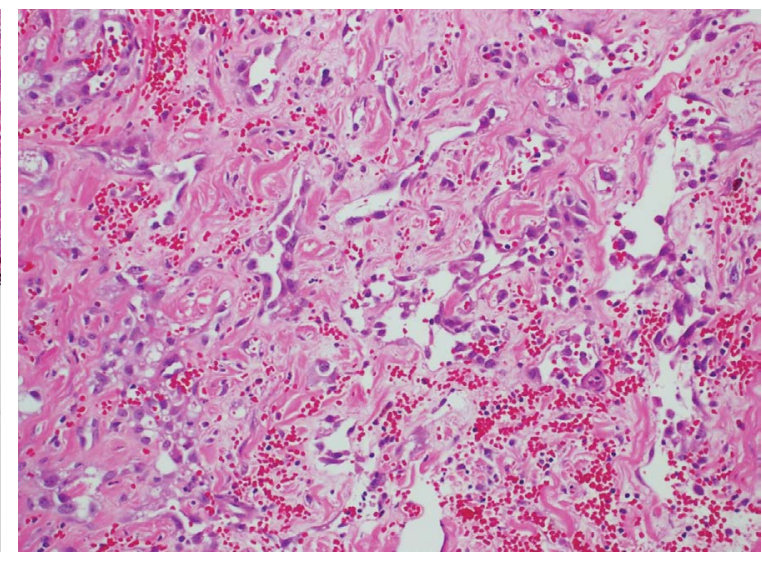

B

Figure 2: A) Biopsy of the scalp consistent with angiosarcoma; B) Histologic specimen viewed at low and high power, respectively.

CD31, a marker recognized as highly sensitive and specific (Figure 2A and Figure 2B). The patient subsequently had multiple scalp excisions approximately $2 \mathrm{~cm}$ from the original site to document the extent of non-clinically visible disease - 5 of which showed atypia concerning for peripheral invasion of the angiosarcoma. Metastatic work-up was negative at this time.

Approximately 1.5 weeks after initial diagnosis, the patient underwent major surgical resection of the scalp tumor and surrounding areas $(12 \mathrm{~cm} \times 12 \mathrm{~cm})$ with split-thickness skin graft reconstruction, harvested from the thigh. He required revision 1 month later due to partial skin graft necrosis, and multiple additional surgical revisions throughout his clinical course to improve wound healing (Figure 3).

While awaiting the initiation of radiation treatment, the patient noticed two new black nodules at the periphery of the surgical scar. These were biopsied and were consistent with recurrent angiosarcoma. He underwent post-op radiation therapy to the entire scalp for 3 months (receiving a total of 72Gy), along with hyperthermia treatments. A specialized technique called

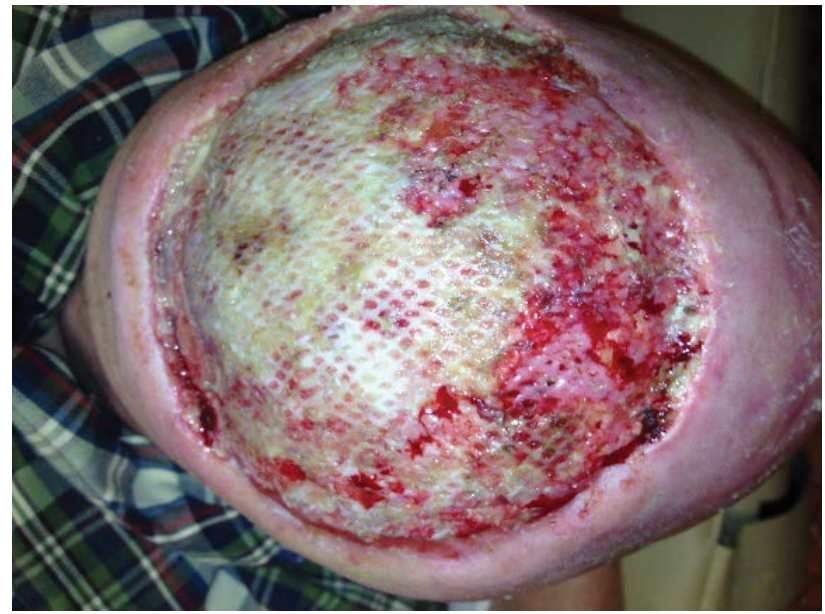

Figure 3: Site of previous angiosarcoma, mid-clinical course, after multiple resections and reconstructions; Skin graft evident.

the "mohawk technique" was used to deliver radiation therapy to the site to ensure adequate coverage of all the suspected areas of persistent disease on the scalp, and to limit dosage to the brain. Due to his recurrent, persistent lesions, the patient then began neoadjuvant 


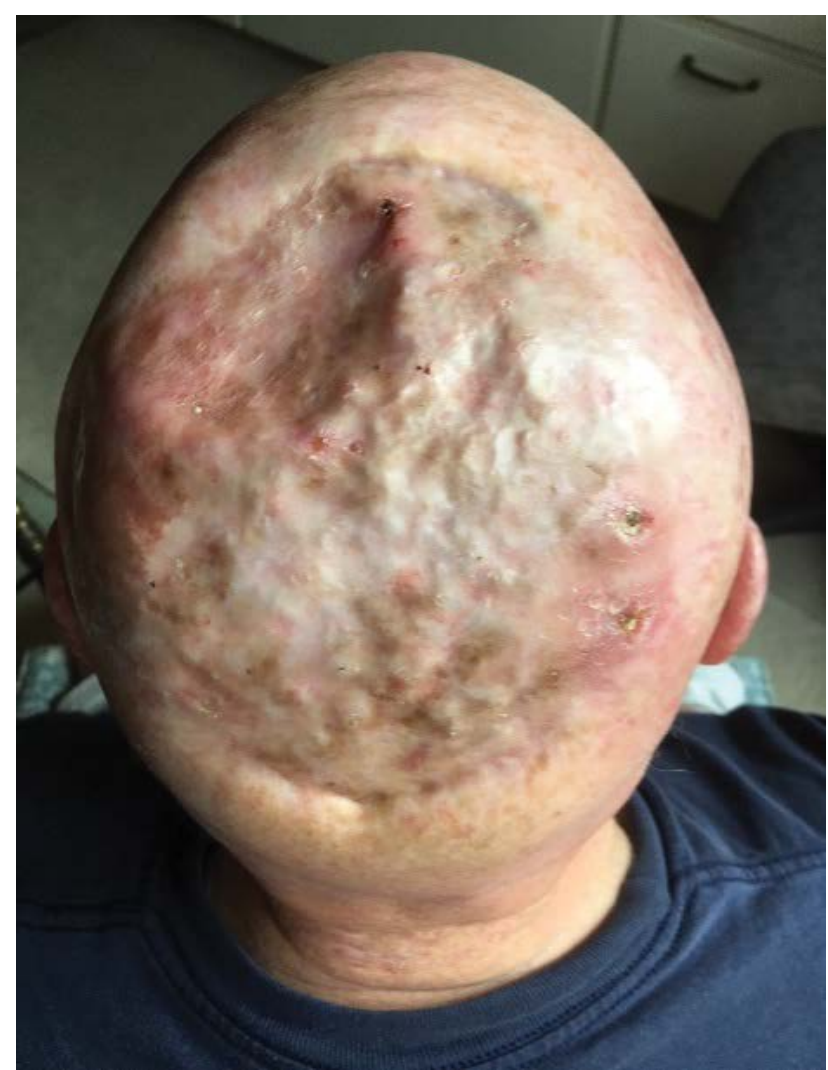

Figure 4: Site of previous angiosarcoma, following radiation therapy, now in remission; 6 years subsequent to his initial diagnosis.

chemotherapy with paclitaxel. He also had subsequent re-excision of the suspicious areas which were concerning for angiosarcoma. The patient did remarkably well after completing his multimodal treatment. He was restarted on low-dose MTX for control of his psoriasis. He had no evidence of recurrence or metastases for approximately 3 years, at which time he noticed an enlarging lymph node in the left supraclavicular space for which FNA and PET scan were abnormal. Eventually, he underwent cervical lymph node dissection which revealed one lymph node to be highly suspicious for metastatic angiosarcoma. He was at this time re-started on paclitaxel and completed a course of 20 treatments. He discontinued treatment 1 month early due to toxicity.

At the time of this article's submission the patient continues in remission without signs of recurrence or evidence of metastases (Figure 4). He is currently 2 years out from lymph node metastasis and 6 years out from initial diagnosis. He reports continued post-chemotherapy neuropathy and chronic positional lymphedema of his lower neck and continues to be seen by dermatology, oncology, and the lymphedema clinic. He is currently maintained on ustekinumab with excellent control of his psoriasis and no evidence of cutaneous or systemic recurrence.

\section{Discussion}

Cutaneous angiosarcoma is a rare sarcoma of endothelial origin which arises most commonly on the head and neck, particularly on the scalp, however it may affect any area of the body. Typically, cutaneous angiosarcoma affects Caucasian males over the age of 60 with a predilection of 2-3: 1 compared to females [2,4-6]. Angiosarcomas account for less than $2 \%$ of all soft-tissue sarcomas, with an incidence of 2-3 per million. The cutaneous form accounts for $28 \%$ of these cases $[7,8]$. Approximately $5 \%$ of all malignant cutaneous tumors are angiosarcomas [9]. Risk factors for angiosarcoma include chronic lymphedema, prior radiation exposure, and immunosuppression (specifically seen after renal transplant). Angiosarcomas are frequently categorized as follows: Primary/idiopathic, soft tissue (uncommonly seen in the deep soft tissues of the lower extremities and abdominal wall; Often in patients with familial syndromes), lymphedema-associated (usually seen in post-mastectomy patients within chronic lymphede$\mathrm{ma}$ ), and radiation-induced angiosarcoma (within or adjacent to the radiation field, commonly seen in breast cancer patients) [10]. Primary/idiopathic is the most common form of cutaneous angiosarcoma, which typically involves the face, neck, and scalp of elderly males [7]. However, the etiology of cutaneous angiosarcoma occurring on the scalp or face, as in our patient, has yet to be elucidated [6].

Many cases of cutaneous angiosarcomas arise in sun-exposed areas [2]. It has been theorized that the vascularity of the head and neck, with its dense arrangement of vessels, may preclude it to oncogenic development in the setting of UV radiation [9]. Excessive UV radiation may be a risk factor, but it cannot be the sole factor, as cutaneous angiosarcomas rarely occur on sun exposed areas other than the face, neck, and scalp. Tumor necrosis factor (TNF)-alpha inhibitors like infliximab have shown mixed evidence in regard to increased risk of overall malignancy stemming from their use. Major observational studies have not confirmed an increased risk. In addition, challenges exist in establishing whether there is true risk between TNF- $\alpha$ inhibitor use and development of malignancy or whether underlying systemic inflammation, other underlying disorders, concomitant medications, or prior history of malignancy contribute to an increased predisposition [11]. Methotrexate has also been implicated in increasing risk via activation of lytic EBV infection, releasing infectious virus into the host cells, allowing the virus to collaborate with the medication's immunosuppressive effects to induce EBV lymphomas in patients with rheumatoid arthritis and polymyositis. These patients are already known to have a higher risk of developing B-cell lymphomas compared to their healthy counterparts [12]. Increasingly, the direct relationship of these medications to an increased risk of malignancy is being questioned. To our knowledge this is the first case report in a patient on a combination of a systemic agent with a biologic drug developing an angiosarcoma. We do believe the effect of these two drugs in combination on his diagnosis remains uncertain. 
Angiosarcomas have been reported in immunosuppressed patients following renal transplants specifically. However, the etiology of angiosarcoma in this setting as in our case also remains unclear. Interestingly, multiple cases of angiosarcoma in renal transplant cases have occurred adjacent to or within the AV fistulas. It is thought that this may be due to turbulent flow at the site combined with long-term immunosuppression which results in the upregulation of growth factors allowing for increased endothelial proliferation and growth of tumor [10].

Angiosarcoma is highly malignant and frequently metastasizes at early stages of the disease. Most commonly, metastasis to the lungs occurs, frequently leading to hemopneumothorax in these patients [13]. Other frequent sites of metastasis include liver, bone, soft tissue, and lymph nodes. Because angiosarcomas can initially resemble benign cutaneous lesions such as ecchymoses or hematomas, diagnosis can be difficult and tends to be delayed, with an average of 5 months from symptom onset to actual diagnosis. As a result of its subtle presentation contributing to a delay in diagnosis and its highly malignant potential, the prognosis for patients diagnosed with angiosarcoma is very poor - with 5 -year disease free survival rates estimated at low as $11 \%$ [7]. Age, primary anatomic site, and extent of disease are the most important prognostic indicators. In an analysis of 434 patients with cutaneous angiosarcomas by Albores-Saveedra, et al., patients had a significantly decreased 10-year survival rate if they were older than 50-years-old compared to patients younger than age 50 ( $37 \%$ vs. $72 \%$, respectively). The tumor of origin's site was also shown to have an impact of survival with 10year survival rates of truncal tumors significantly better than those of the head and neck ( $75 \%$ vs. $14 \%$ respectively). When an angiosarcoma remains localized to the skin, patients exhibit a $54 \%$ survival rate at 10 years [14].

As the disease is rare, treatment guidelines for angiosarcoma have not been standardized, but it continues to be widely accepted that surgery with wide excision of the lesion is the most reliable treatment for cure. Since completely negative margins can be increasingly difficult to achieve surgically and to assess histologically, adjuvant radiation therapy is frequently employed, which has been shown to improve survival time fourfold $[4,5]$. Angiosarcomas pose a surgical challenge since they commonly occur in regions where aggressive surgical excisions can lead to disfigurement, functional impairment, and substantial difficulty in reconstruction. Effective treatment for many patients is hard to achieve since angiosarcomas are frequently large and ill-defined, with a noncontiguous growth pattern often made up of several closely related tumors $[2,7]$. Due to the extent of disease and the impossibility of repair and reconstruction, this can lead to potentially inoperable tumors and poorer outcomes. Primary closures are almost always impossible. As such, combination therapy is con- sidered the best option to optimize a patient's survival.

Because of the rarity of angiosarcomas, studies regarding the molecular mechanisms and pathogenesis of the disease are rare. Angiogenic factors are thought to play a large role in angiosarcoma development. Vascular endothelial growth factor (VEGF) and its receptors are overexpressed in $80 \%$ of angiosarcomas [15]. A study of 19 cases of angiosarcomas explored the hypothesis that the p53/murine double minus-2 protein (MDM-2) pathway plays a key role in angiosarcoma development. More than two thirds of the angiosarcomas analyzed showed dysfunction of the p53/MDM-2 pathway. This pathway affects both cellular growth and angiogenesis in addition to up-regulation of VEGF itself, leading to higher concentrations of VEGF and decreased thrombospondin 1 (an antiangiogenic factor) [16]. Not surprisingly, targeted therapy with inhibitors of VEGF, such as bevacizumab, have shown some efficacy in treatment when used in combination with radiotherapy $[2,8]$. In one study, bevacizumab treatment showed stabilization of disease or partial response in $11 / 23$ patients [2]. Reports of remission after combined use of adjuvant radiation therapy and bevacizumab followed by surgery have also been described $[8,17]$. In addition, a case of cutaneous angiosarcoma treated with a combination of paclitaxel and bevacizumab without radiotherapy showed significant improvement [15].

As cutaneous angiosarcoma expresses a high level of VEGF, there is evidence that taxane-based chemotherapeutic regimens may improve survival and may even be efficacious as first-line therapy for angiosarcoma since they are anti-angiogenic in addition to having a direct anti-tumor effect $[18,19]$. One phase 2 trial of paclitaxel revealed $17 \%$ of patients as responders to treatment, with two of these patients achieving complete response subsequent to surgery [20]. Anti-VEGF pathway therapy with drugs like sorafenib and pazopanib have also shown effectiveness [13,21-23]. A phase II trial using a programmed cell death protein (PD-1) inhibitor for soft-tissue and bone sarcomas along with a few case reports regarding cutaneous angiosarcoma have shown response to PD-1 inhibitor therapy [24-26]. The use of adjuvant chemotherapy for treatment of angiosarcoma continues to be controversial. It is usually considered as a treatment option when patients present with metastatic disease or for palliation of locally advanced disease [4].

Early diagnosis of angiosarcoma is important as localized and systemic spread occurs rapidly and rates of early metastasis are high. Clinicians should strive for early diagnosis, early and aggressive resection, and a multidisciplinary treatment approach to improve disease control and patient outcomes. Lifelong follow up is indicated in these patients as local recurrence rates have been observed in $35-86 \%$ of patients, depending on the approach to treatment [5]. 


\section{Conclusion}

The six-year survival of this 66-year-old male following both local recurrence of his angiosarcoma, as well as a single lymph node metastasis, is highly favorable. Whether infliximab +/- methotrexate contributed to the development of cutaneous angiosarcoma in this patient, or if it was imminent on a background of chronic psoriatic inflammation and ulceration, may be better answered through a prospective cohort study which could reveal information unbeknown to physicians, that they may need to use caution in approved practice and carefully survey these patients for signs and symptoms of angiosarcoma. It remains uncertain whether the patient's excess of 10-year therapy with a combination of infliximab and methotrexate for generalized plaque psoriasis played a role in his aggressive scalp angiosarcoma.

\section{Funding Sources}

None.

\section{References}

1. Hata M (2018) Radiation therapy for angiosarcoma of the scalp: Total scalp irradiation and local irradiation. Anticancer Res 38: 1247-1253.

2. Spiker AM, Ramsey ML (2018) Cancer, angiosarcoma. Stat pearls publishing.

3. Young RJ, Brown NJ, Reed MW, Hughes D, Woll PJ (2010) Angiosarcoma. Lancet Oncol 11: 983-991.

4. Kohen D, Dross P (2013) Angiosarcoma of the scalp. Delaware Medical Journal 85: 269-272.

5. Pawlik TM, Paulino AF, McGinn CJ, Baker LH, Cohen DS, et al. (2003) Cutaneous angiosarcoma of the scalp: A multidisciplinary approach. Cancer 98: 1716-1726.

6. Dhanasekar P, Karthikeyan VS, Rajkumar N, Chandra Sistla S, Manwar Ali S, et al. (2012) Cutaneous angiosarcoma of the scalp masquerading as a squamous cell carcinoma: Case report and literature review. J Cutan Med Surg 16: 187-190.

7. Oashi K, Namikawa K, Tsutsumida A, Takahashi A, Itami $J$, et al. (2018) Surgery with curative intent is associated with prolonged survival in patients with cutaneous angiosarcoma of the scalp and face-a retrospective study of 38 untreated cases in the Japanese population. Eur J Surg Oncol 44: 823-829.

8. Koontz BF, Miles EF, Rubio MA, Madden JF, Fisher SR, et al. (2008) Preoperative radiotherapy and bevacizumab for angiosarcoma of the head and neck: Two case studies. Head Neck 30: 262-266.

9. Rouhani P, Fletcher CD, Devesa SS, Toro JR (2008) Cutaneous soft tissue sarcoma incidence patterns in the U.S.: An analysis of 12,114 cases. Cancer 113: 616-627.

10. Ahmed I, Hamacher KL (2002) Angiosarcoma in a chronically immunosuppressed renal transplant recipient: Report of a case and review of the literature. Am J Dermatopathol 24: $330-335$.

11. Askling J, van Vollenhoven RF, Granath F, Raaschou P, Fored CM, et al. (2009) Cancer risk in patients with rheumatoid arthritis treated with anti-tumor necrosis factor alpha therapies: Does the risk change with the time since start of treatment? Arthritis Rheum 60: 3180-3189.
12. Feng WH, Cohen JI, Fischer S, Li L, Sneller M, et al. (2004) Reactivation of latent epstein-barr virus by methotrexate: A potential contributor to methotrexate-associated lymphomas. J Natl Cancer Inst 96: 1691-1702.

13. Wada N, Uchi H, Furue M (2018) Case of angiosarcoma of the scalp successfully controlled by eribulin. J Dermatol 45: $116-117$

14. Albores-Saavedra J, Schwartz AM, Henson DE, Kostun L, Hart A, et al. (2011) Cutaneous angiosarcoma. Analysis of 434 cases from the surveillance, epidemiology, and end results program, 1973-2007. Ann Diagn Pathol 15: 93-97.

15. Fuller CK, Charlson JA, Dankle SK, Russell TJ (2010) Dramatic improvement of inoperable angiosarcoma with combination paclitaxel and bevacizumab chemotherapy. J Am Acad Dermatol 63: e83-e84.

16. Zietz C, Rössle M, Haas C, Sendelhofert A, Hirschmann A, et al. (1998) MDM-2 Oncoprotein overexpression, p53 gene mutation, and vegf up-regulation in angiosarcomas. Am J Pathol 153: 1425-1433.

17. Gonzalez MJ, Koehler MM, Satter EK (2009) Angiosarcoma of the scalp: A case report and review of current and novel therapeutic regimens. Dermatol Surg 35: 679-684.

18. Belotti D, Vergani V, Drudis T, Borsotti P, Pitelli MR, et al. (1996) The microtubule-affecting drug paclitaxel has antiangiogenic activity. Clin Cancer Res 2: 1843-1849.

19. Seonggyu Byeon, Haa-Na Song, Hee Kyung Kim, Jun Soo Ham, Su Jin Lee, et al. (2016) A Korean single-center, real-world, retrospective study of first-line weekly paclitaxel in patients with metastatic angiosarcoma. Clinical Sarcoma Research 6: 8.

20. Penel N, Bui BN, Bay JO, Cupissol D, Ray-Coquard I, et al. (2008) Phase II trial of weekly paclitaxel for unresectable angiosarcoma: The angiotax study. J Clin Oncol 26: 52695274.

21. Fata F, O'Reilly E, Ilson D, Pfister D, Leffel D, et al. (1999) Paclitaxel in the treatment of patients with angiosarcoma of the scalp or face. Cancer 86: 2034-2037.

22. Ray-Coquard I, Italiano A, Bompas E, Le Cesne A, Robin YM, et al. (2012) Sorafenib for patients with advanced angiosarcoma: A phase II trial from the french sarcoma group (GSF/GETO). Oncologist 17: 260-266.

23. van der Graaf WT, Blay JY, Chawla SP, Kim DW, Bui-Nguyen B, et al. (2012) Pazopanib for metastatic soft-tissue sarcoma (PALETTE): A randomized, double-blind, placebo-controlled phase 3 trial. Lancet 379: 1879-1886.

24. Tawbi HA, Burgess M, Bolejack V, Van Tine BA, Schuetze SM, et al. (2017) Pembrolizumab in advanced soft-tissue sarcoma and bone sarcoma (SARC028): A multicentere, two-cohort, single-arm, open-label, phase 2 trial. Lancet Oncol 18: 1493-1501.

25. Sindhu S, Gimber L, Cranmer L, McBride A, Kraft A (2017) Angiosarcoma treated successfully with anti-PD-1 therapy-a case report. J Immunother Cancer 5: 58.

26. Hofer S, Zeidler K, Schipf A, Kempf W, Zimmermann D, et al. (2018) Angiosarcoma of the scalp responding to nivolumab: A case report. Br J Dermatol 179: 530-531. 